\title{
Efeitos da Intervenção Facilitatória na Aquisição de Habilidades Funcionais em Crianças com Paralisia Cerebral
}

\author{
Márcia Midori Morimoto ${ }^{1}$, Cristina dos Santos Cardoso de Sá2, Odete de Fátima Sallas Durigon ${ }^{3}$
}

\section{RESUMO}

Contexto: A Encefalopatia Crônica Não Progressiva da Infância (ECNPI) ou Paralisia Cerebral é uma seqüela de agressão encefálica caracterizada por transtorno motor não evolutivo quanto à sua lesão. O tratamento fisioterápico clássico baseia-se no método Bobath, sendo contra-indicado procedimentos que envolvam padrões reflexos e carga. Objetivo: Estudar o processo de aquisição de habilidades motoras como: sedestação, quadrupedia, engatinhar, ajoelhado, semi-ajoelhado, bipedestação e marcha, em crianças com Paralisia Cerebral sob procedimento facilitatório. Método: Utilizamos o delineamento de sujeito único aplicado a duas crianças com ECNPI, tipo diparesia espástica, entre 0 e 2 anos. Foram realizadas 20 sessões fisioterápicas onde aplicou-se métodos facilitatórios e verificou-se 0 tono muscular e as atividades funcionais estáticas e dinâmicas. Resultados: Em ambos os casos, houve incremento do controle motor nas atividades funcionais estáticas e dinâmicas, e diminuição do tono muscular. Conclusão: A facilitação Neuromuscular Proprioceptiva, deve ser considerada como opção no tratamento da Paralisia Cerebral.

Descritores: Fisioterapia, Paralisia cerebral, Facilitação neuromuscular proprioceptiva, Desenvolvimento motor

\section{SUMMARY}

Backgroundy: The Cerebral Palsy it's a consequence characterized by non-progressive motor disturbance refering to his lesion. The classic physical therapy approach is based on Bobath method, where the procedures that involve reflex and load patterns are non indicated. Objective: To study the acquirement process of motor abilities in children with Cerebral Palsy under facilitatory procedure. Method: On 2 children with Cerebral Palsy, spastic diparetic type, between 0 and 2 years old. Twenty physical therapy sessions were realized where facilitatory method were applied. The muscular tone and the static and dynamics functional activities were examinated. Results: In both cases, an improve of motor control in static and dynamics functional activities and muscular tone decrease had occurred. Conclusion: The Proprioceptive Neuromuscular Facilitation procedure has to be considered as an option on the Cerebral Palsy treatment.

Keywords: Physical therapy, Cerebral palsy, Proprioceptive neuromuscular facilitation, Motor development

\section{INTRODUÇÃO}

Encefalopatia Crônica Não Progressiva da Infância (ECNPI) ou Paralisia Cerebral (PC) é uma seqüela de uma agressão encefálica, que se caracteriza primordialmente por um transtorno persistente, porém não invariável do tono, da postura e do movimento, que surge na primeira infância e que não é somente secundária a esta lesão não evolutiva do encéfalo, mas se deve também à influência que a referida lesão exerce sobre a maturação neurológica'.

$\mathrm{Na}$ ECNPI, a lesão interfere no desenvolvimento ordenado, pois sabe-se que crianças normais durante o primeiro ano de vida apresentam inicialmente uma exuberância de projeções Ia e de motoneurônios a que pas- sam por uma diminuição gradual, assim a intensidade da resposta reflexa sofre alterações durante o desenvolvimento. Em lesões corticais ou subcorticais, pode ocorrer retenção desta exuberância sináptica, que associados com a deficiência de entradas supraespinais que deprimem a atividade reflexa, contribuem para a alteração do equilíbrio fisiológico da inibição recíproca. Resultando, assim, na modulação inadequada dos reflexos e das reações de endireitamento, que são importantes no desenvolvimento do controle tônico e postural que iniciam-se pelo controle de cabeça impedindo a criança de rolar da posição supina para a posição prona, e mais tarde de assumir a sedestação a partir da posição prona ${ }^{(2,3)}$

Trabalho realizado na Faculdade de Medicina da Universidade de São Paulo - FMUSP

1 - Supervisora de Estágio do Curso de Fisioterapia da FMUSP, Mestranda em Neurociências e Comportamento

2 - Profa. do Curso de Fisioterapia do IMES, Doutoranda em Neurociências e Comportamento USP

3 - Profa. Dra. do Curso de Fisioterapia da USP

Endereço para correspondência: Curso de Fisioterapia Da Faculdade De Medicina Da Universidade De São Paulo. Rua Cipotânia, 51

Cidade Universitária - 05360-000 São Paulo - SP. 
A reabilitação se apoia no objetivo de mudar a resposta de uma criança com lesão cerebral para uma semelhante a de uma criança normal, baseados na capacidade que aquela tem de adquirir novas respostas à estimulação ${ }^{4}$. Há numerosos métodos utilizados para tratamento da ECNPI, sendo a abordagem fisioterápica clássica o método Bobath ${ }^{5}$, baseado na facilitação motora por inibição de padrões tônicos patológicos através de pontos chaves de controle. Apesar de haverem poucos estudos comprovando a eficácia das técnicas fisioterápicas no tratamento da criança com ECNPI de forma criteriosa ${ }^{6}$ costuma-se desaconselhar a utilização de outras formas de intervenção, especialmente aquelas que envolvem padrões reflexo e carga, baseados na observação de ocorrência de aumentos transitórios do tono muscular e de reações associadas. Pouco se conhece sobre o processo de aquisição sob um tratamento de facilitação baseada no princípio de somação espacial de sinapses nervosas e na somação temporal das mesmas. "Facilitação refere-se a uma capacidade aumentada em iniciar uma resposta motora através de um aumento da atividade neuronal, e de um potencial sináptico alterado."7

O objetivo de nosso trabalho foi estudar o processo de aquisição de habilidades motoras como: sedestação, quadrupedia, engatinhar, ajoelhado, semi-ajoelhado, bipedestação e marcha, em crianças com encefalopatia crônica não progressiva da infância sob procedimento terapêutico facilitatório.

\section{METODOLOGIA}

Participaram deste estudo duas crianças com diagnóstico de ECNPI espástica diparética, entre 0 a 2 anos, sob análise de sujeito único. A intervenção fisioterápica baseou-se nas técnicas de facilitação neuromuscular proprioceptiva (FNP) e os dados avaliados foram tono muscular através da escala de avaliação do tono muscular desenvolvida por Durigon e Piemonte ${ }^{8}$ em adultos, e validada para crianças com $\mathrm{ECNPI}^{9}$, e as atividades funcionais estáticas e dinâmicas através da escala de avaliação de atividades funcionais desenvolvida por Durigon, Sá \& Sita $^{10}$.

A linha de base consistiu em uma avaliação inicial realizada em duas sessões em que foram aplicados os protocolos de avaliação neurológica, avaliação do tono muscular e avaliação da atividade funcional. Foram realizadas duas sessões fisioterápicas semanais com duração de 45 minutos por um período de 10 semanas, totalizando 20 sessões. Os protocolos de tono muscular e atividade funcional foram aplicados a cada 10 sessões.

A intervenção fisioterápica foi realizada através da técnica de F.N.P. que envolveu os seguintes procedimentos: exercícios em padrão diagonal de membros, tronco e cabeça de forma enfatizando-se a técnica de iniciação rítmica associadas ao estiramento muscular, tração e/ou aproximação e resistência; exercícios com a técnica de estabilização rítmica; a utilização de reflexos primários na aquisição e manutenção das posturas (progressão em prono e supino de acordo com a necessidade de cada paciente), assim como para a realização do engatinhar e da marcha; foram exploradas as reações posturais e de equilíbrio; e exercícios de alongamento no padrão diagonal.

\section{RELATO DE CASO CLÍNICO}

\section{CASO CLÍNICO I}

Paciente, sexo feminino, com 8 meses de idade corrigida, parda, recém-nascida pré-termo de 7 meses e de parto cesárea, com diagnóstico clínico de ECNPI devido anóxia cerebral grave em decorrência de sofrimento fetal agudo, com conseqüente quadro de desconforto respiratório que regrediu com a ventilação mecânica. A criança permaneceu na UTI durante 2 meses e 5 dias onde apresentou várias complicações como: icterícia, anemia, broncopneumonia, laringite pós-extubação e hidrocefalia comunicante com hipertensão intra-craniana evoluindo com aumento do perímetro cefálico sendo necessário a colocação de derivação interna ventrículo-peritoneal permanente. A paciente foi encaminhada à fisioterapia no Centro da Docência e Pesquisa em Fisioterapia da USP em fevereiro de 1997. A avaliação inicial, revelou quadro clínico de diparesia espástica. As atividades funcionais e performance motora, como as estáticas (sedestação, quadrupedia, ajoelhado, semi-ajoelhado e bipedestação) apresentavam-se comprometidas assim como as dinâmicas (engatinhar e marcha).

A hipertonia foi quantificada e os dados podem ser observados no Quadro 1, onde verifica-se distribuição simétrica do tono bilateralmente em padrão flexor de membros superiores (MMSS) e em padrão extensor de MMII (MMII), sendo este o mais predominante.

A performance motora estática e funcional também foram quantificadas e podem ser observadas nos Quadros 2 e 3 respectivamente.

Ao avaliar a sedestação, esta foi classificada com o grau 4 pois a paciente era capaz de assumir a postura completa somente com auxílio do terapeuta, mantinha a postura sem apoio e com bom alinhamento, apesar de deslocar o peso sobre o osso sacro e anteriorizar o tronco. Apresentava reação de proteção anterior, látero-lateral e posterior com bom equilíbrio e controle de tronco. Há a presença também de adução escapular para auxiliar a evocação do tono extensor, o que pode acarretar um bloqueio escapular limitando a funcionalidade de MMSS e o retardo das reações de proteção. A postura quadrúpede foi classificada com grau 8 pois a criança a assumia sem auxílio a partir da sedestação, mantinha sem apoio e com alinhamento, distribuição de peso simétrica e sem aumento de base, porém ao manipular objetos com um dos MMSS observava-se discreto desalinhamento de tronco. As posturas intermediárias foram classificadas com grau 2, significando que adotava a posição com auxílio de MMSS apoiados em uma superfície mais alta, a mantinha somente com apoio de MMSS e sem alinhamento. A bipedestação foi classificada com grau 3 pois a paciente assume a postura com apoio de MMSS em uma superfície mais alta a partir da quadrupedia, passando pelo ajoelhado e semi ajoelhado. Permanece na postura bípede sem apoio por curto período de tempo com oscilação de tronco superior e inferior, necessitando depois de apoio de MMSS. Nesta posição, não verifica-se a presença do reflexo de apoio positivo, porém observa-se aumento da base de sustentação, semiflexão de quadril e aumento da lordose lombar.

O engatinhar foi realizado em padrão normal e coor- 
QUADRO 1 - CLASSIFICAÇÃO DO TONO MUSCULAR DO CASO CLÍNICO I E II

\begin{tabular}{|c|c|c|c|c|c|c|c|c|c|c|c|c|c|}
\hline \multirow{2}{*}{\multicolumn{2}{|c|}{\begin{tabular}{|c|} 
GRUPO \\
MUSCULAR
\end{tabular}}} & \multicolumn{6}{|c|}{ CASO CLÍNICOI } & \multicolumn{6}{|c|}{ CASO CLÍNICO II } \\
\hline & & \multicolumn{2}{|c|}{$\begin{array}{l}\text { AVALIAÇÃO } \\
\text { INICIAL }\end{array}$} & \multicolumn{2}{|c|}{$\begin{array}{c}10^{\mathrm{a}} \\
\text { TERAPIA }\end{array}$} & \multicolumn{2}{|c|}{$\begin{array}{c}20^{\mathrm{a}} \\
\text { TERAPIA }\end{array}$} & \multicolumn{2}{|c|}{$\begin{array}{l}\text { AVALIAÇÃO } \\
\text { INICIAL }\end{array}$} & \multicolumn{2}{|c|}{$\begin{array}{c}10^{\mathrm{a}} \\
\text { TERAPIA }\end{array}$} & \multicolumn{2}{|c|}{$\begin{array}{c}20 \\
\text { TERAPIA }\end{array}$} \\
\hline & & $\mathrm{E}$ & D & $E$ & D & $\mathrm{E}$ & D & $E$ & D & $\mathrm{E}$ & $\mathrm{D}$ & $\mathrm{E}$ & D \\
\hline \multirow[t]{6}{*}{ Ombro } & Flexor & 3 & 3 & 3 & 3 & 3 & 3 & 2 & 2 & 2 & 2 & 2 & 2 \\
\hline & Adutor & 3 & 3 & 3 & 3 & 3 & 3 & 2 & 2 & 2 & 2 & 2 & 2 \\
\hline & Rotador Medial & 3 & 3 & 3 & 3 & 2 & 2 & 2 & 2 & 2 & 2 & 2 & 2 \\
\hline & Extensor & 2 & 2 & 2 & 2 & 2 & 2 & 2 & 2 & 2 & 2 & 2 & 2 \\
\hline & Abdutor & 2 & 2 & 2 & 2 & 2 & 2 & 2 & 2 & 2 & 2 & 2 & 2 \\
\hline & Rotador Lateral & 2 & 2 & 2 & 2 & 2 & 2 & 2 & 2 & 2 & 2 & 2 & 2 \\
\hline \multirow[t]{2}{*}{ Cotovelo } & Flexor & 3 & 3 & 3 & 3 & 3 & 3 & 3 & 2 & 3 & 2 & 3 & 2 \\
\hline & Extensor & 2 & 2 & 2 & 2 & 2 & 2 & 2 & 2 & 2 & 2 & 2 & 2 \\
\hline \multirow[t]{2}{*}{ Punho } & Flexor & 2 & 2 & 2 & 2 & 2 & 2 & 3 & 2 & 3 & 2 & 2 & 2 \\
\hline & Extensor & 2 & 2 & 2 & 2 & 2 & 2 & 2 & 2 & 2 & 2 & 2 & 2 \\
\hline \multirow[t]{2}{*}{ Dedos } & Flexor & 2 & 2 & 2 & 2 & 2 & 2 & 3 & 2 & 3 & 2 & 3 & 2 \\
\hline & Extensor & 2 & 2 & 2 & 2 & 2 & 2 & 2 & 2 & 2 & 2 & 2 & 2 \\
\hline \multirow[t]{6}{*}{ Quadril } & Extensor & 6 & 6 & 6 & 6 & 2 & 4 & 2 & 2 & 2 & 2 & 2 & 2 \\
\hline & Abdutor & 2 & 2 & 2 & 2 & 2 & 2 & 2 & 2 & 2 & 2 & 2 & 2 \\
\hline & Rotador Lateral & 2 & 2 & 2 & 2 & 2 & 2 & 2 & 2 & 2 & 2 & 2 & 2 \\
\hline & Flexor & 2 & 2 & 2 & 2 & 2 & 2 & 2 & 2 & 2 & 2 & 2 & 2 \\
\hline & Adutor & 3 & 3 & 3 & 3 & 3 & 3 & 3 & 3 & 3 & 3 & 3 & 3 \\
\hline & Rotador Medial & 3 & 3 & 3 & 3 & 3 & 3 & 3 & 3 & 3 & 3 & 3 & 3 \\
\hline \multirow[t]{2}{*}{ Joelho } & Flexor & 2 & 2 & 2 & 2 & 2 & 2 & 2 & 2 & 2 & 2 & 2 & 2 \\
\hline & Extensor & 6 & 6 & 6 & 6 & 6 & 6 & 6 & 3 & 6 & 3 & 6 & 3 \\
\hline \multirow[t]{2}{*}{ Tornozelo } & Flexor & 2 & 2 & 2 & 2 & 2 & 2 & 2 & 2 & 2 & 2 & 2 & 2 \\
\hline & Extensor & 6 & 6 & 6 & 6 & 6 & 6 & 6 & 6 & 6 & 6 & 6 & 6 \\
\hline
\end{tabular}

FONTE: DURIGON, O.F.S., PIEMONTE, M.E.P. Desenvolvimento de protocolo para avaliação de hipertonia, 1993.

QUADRO 2- CLASSIFICAÇÃO DAS ATIVIDADES FUNCIONAIS ESTÁTICAS DO CASO CLÍNICO I E II

\begin{tabular}{|c|c|c|c|c|c|c|}
\hline \multirow[b]{2}{*}{ POSIÇÃO } & \multicolumn{3}{|c|}{ CASO CLÍNICO I } & \multicolumn{3}{|c|}{ CASO CLÍNICO II } \\
\hline & AVALIAÇÃOINICIAL & 10'TERAPIA & 20TERAPIA & AVALIAÇÃOINICIAL & $10^{2}$ TERAPIA & 20?TERAPIA \\
\hline Sedestação & 4 & 9 & 9 & 7 & 7 & 8 \\
\hline Quadrupedia & 8 & 9 & 9 & 8 & 8 & 8 \\
\hline Ajoelhado & 2 & 2 & 5 & 1 & 2 & 7 \\
\hline Semi-ajoelhado & 2 & 2 & 5 & 1 & 1 & 2 \\
\hline Bipedestação & 3 & 4 & 9 & 1 & 2 & 7 \\
\hline
\end{tabular}

FONTE: DURIGON, O.F.S.; SÁ, C.S.C.; SITA, L.V. Desenvolvimento de um protocolo de avaliação do desempenho motor e funcional em crianças com ECNPI, 1996

QUADRO 3- CLASSIFICAÇÃO DAS ATIVIDADES FUNCIONAIS DINÂMICAS DO CASO CLÍNICO I E II

\begin{tabular}{|c|c|c|c|c|c|c|}
\hline \multirow[b]{2}{*}{ POSIÇÃO } & \multicolumn{3}{|c|}{ CASO CLÍNICO I } & \multicolumn{3}{|c|}{ CASO CLÍNICO II } \\
\hline & AVALIAÇÃOINICIAL & 10'TERAPIA & $20^{2}$ TERAPIA & AVALIAÇÃOINICIAL & 10TERAPIA & 20TERAPIA \\
\hline Engatinhar & 2 & 3 & 3 & 3 & 3 & 3 \\
\hline Marcha & 0 & 1 & 3 & 0 & 1 & 1 \\
\hline
\end{tabular}

FONTE: DURIGON, O.F.S.; SÁ, C.S.C.; SITA, L.V. Desenvolvimento de um protocolo de avaliação do desempenho motor e funcional em crianças com ECNPI, 1996 


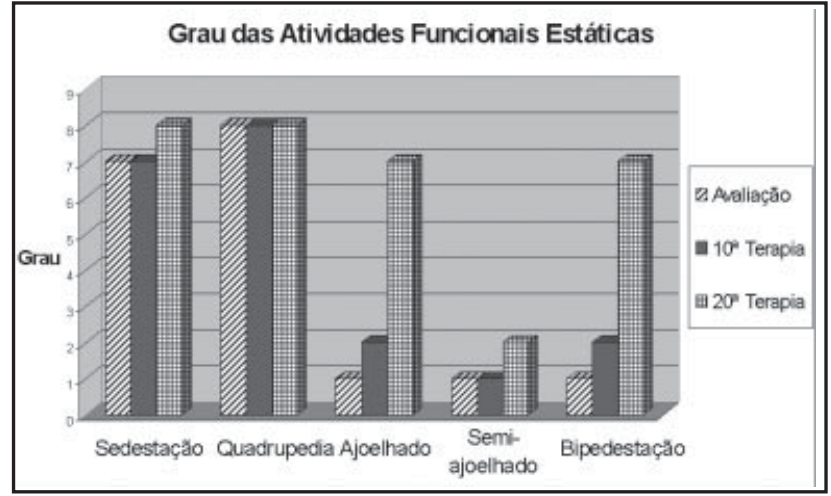

Figura 1 - Evolução das atividades funcionais estáticas após tratamento fisioterápico

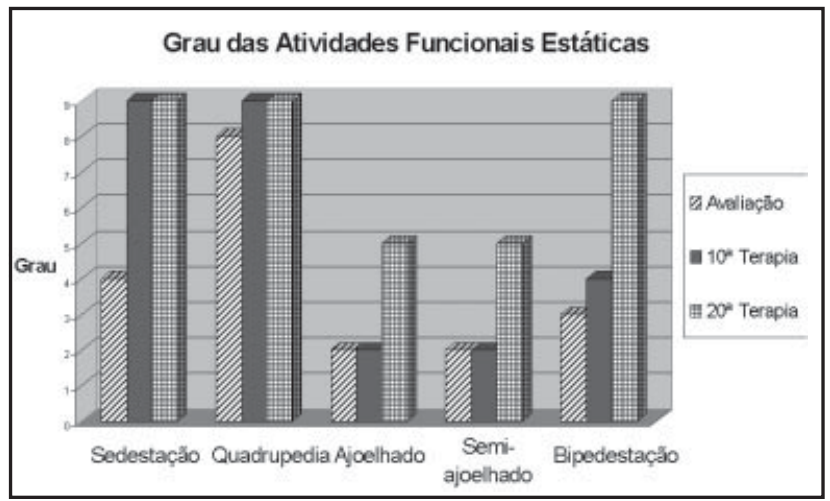

Figura 3 - Evolução das atividades funcionais estáticas após tratamento fisioterápico

denado porém com interferência da hipertonia, apresentando diminuição do controle de tronco inferior sendo classificada então com grau 2. A criança não realiza a marcha mesmo com apoio da terapeuta em quadril ou a apoiando pelos MMSS, sendo então classificada com grau 0.

A estratégia terapêutica utilizada foi primeiramente a aquisição da estabilidade de tronco inferior e superior nas diversas posturas associadas ao ganho de equilíbrio e força muscular (músculos abdominais e extensores de tronco inferior), treino das passagens das posturas (progressão em prono e supino) até atingir a bipedestação. Objetivou-se também evocar a motricidade voluntária de MMII para o treino de marcha. A conduta foi baseada nos recursos da técnica terapêutica da FNP.

\section{CASO CLÍNICO II}

Paciente, sexo feminino, com 1 ano e 2 meses de idade corrigida, parda, recém-nascida pré-termo de 7 meses e de parto normal, com diagnóstico clínico de ECNPI devido anóxia cerebral grave em decorrência de sofrimento fetal agudo, com conseqüente quadro de desconforto respiratório permanecendo 30 dias na UTI. sob ventilação mecânica por cânula oro-traqueal, passando em seguida para um berçário de médio risco onde passou mais 30 dias, tendo alta a seguir. Durante a internação hospitalar apresentou intercorrências como alguns períodos de taquicardia. A família da paciente foi encaminhada à fisioterapia no Centro da Docência e Pesquisa em Fisioterapia da USP. A queixa principal da família era a não deambulação da paciente.

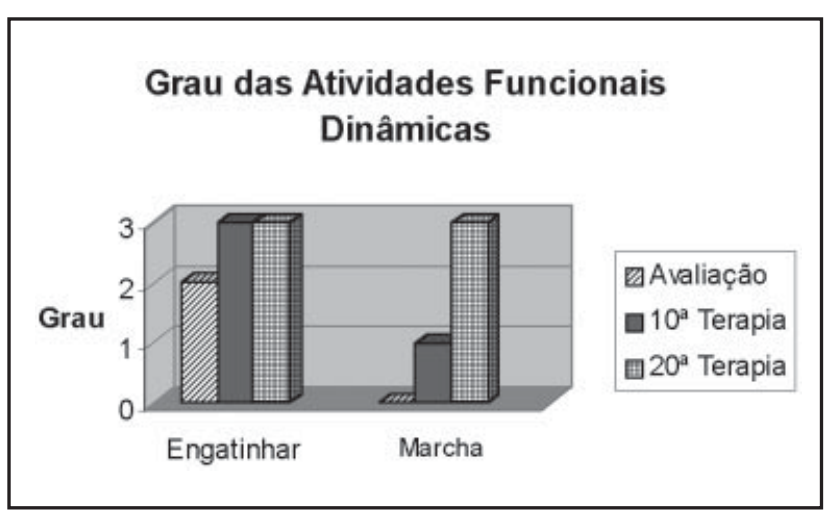

Figura 2 - Evolução das atividades funcionais dinâmicas após tratamento fisioterápico

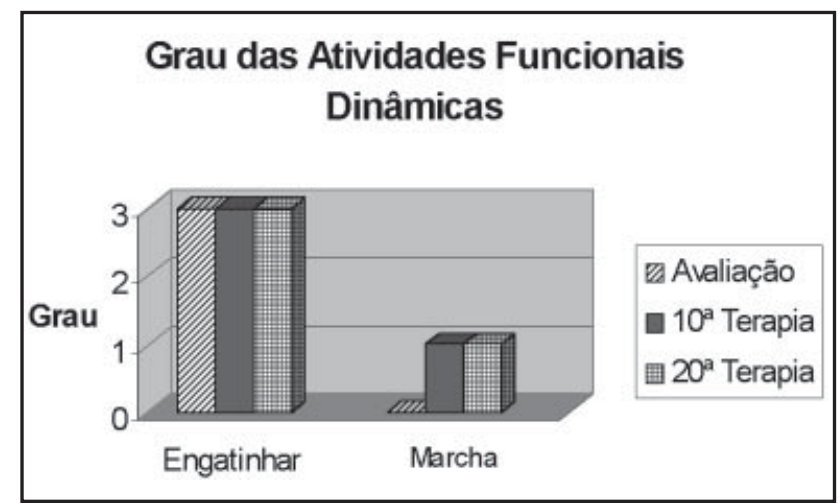

Figura 4 - Evolução das atividades funcionais dinâmicas após tratamento fisioterápico

A avaliação inicial revelou quadro clínico de diparesia espástica com predomínio em hemicorpo esquerdo sem déficits sensitivos. As atividades funcionais e performance motora, como as estáticas (ajoelhado, semi-ajoelhado e bipedestação) apresentavam-se comprometidas assim como as dinâmicas (marcha). A hipertonia foi quantificada e os dados podem ser observados no Quadro 1, onde verifica-se distribuição assimétrica do tono bilateralmente em padrão flexor de MMSS e em padrão extensor de MMII, sendo a hipertonia predominante em MMII e em hemicorpo esquerdo.

A performance motora estática e funcional também foram quantificadas e podem ser observadas nos Quadros 2 e 3 respectivamente.

Ao avaliar a sedestação, esta foi classificada com o grau 7 significando que a paciente era capaz de assumir a postura sem auxílio, a mantinha sem apoio com a base de sustentação aumentada, mas não possuía um bom alinhamento pois o tronco encontrava-se anteriorizado e lateralizado para a direita com a cabeça protraída, desloca o peso sobre o osso sacro e freqüentemente realizava apoio com o membro superior direito. Apresentava reação de equilíbrio deficitária. Já nesta postura é possível observar importante hipertonia extensora de tornozelos acompanhada de eversão principalmente em membro inferior esquerdo. A postura quadrúpede foi classificada com grau 8 pois a criança a assumia sem auxílio a partir da sedestação realizando dissociação de cinturas, mantinha sem apoio com base de sustentação aumentada e com alinhamento, porém ao manipular objetos com um 
dos MMSS observava-se desalinhamento de tronco. As posturas intermediárias foram classificadas com grau 1, significando que adotava a posição com auxílio de MMSS apoiados em uma superfície mais alta, a mantinha somente com apoio de MMSS com semi-flexão acentuada de joelho e quadril, principalmente de membro inferior esquerdo, e sem alinhamento. A bipedestação foi classificada com grau 1 pois a paciente assumiu a postura com apoio de MMSS em uma superfície mais alta a partir da quadrupedia, passando pelo ajoelhado e semi ajoeIhado. Manteve a postura com apoio de MMSS, aumento da base de sustentação e sem alinhamento, onde verifica-se semiflexão de joelhos, extensão de tornozelos e eversão de pés em ambos os MMII sendo mais acentuados no esquerdo. A descarga de peso é realizada, então, em hemicorpo direito. Nesta posição, verifica-se a presença do reflexo de apoio positivo.

O engatinhar foi realizado em padrão normal e coordenado sem interferência da hipertonia, sendo classificada então com grau 3. A criança não realiza a marcha sem apoio mesmo com apoio da terapeuta em quadril, sendo então classificada com grau 0 .

A estratégia terapêutica utilizada foi a aquisição de posturas mais altas como a bipedestação e a realização da marcha através do controle de tronco inferior estático e dinâmico, treinando-se estabilidade, equilíbrio, fortalecimento de músculos extensores lombares e abdominais, treino de passagem das diversas posturas e treino de marcha através da técnica de FNP.

\section{RESULTADOS}

\section{CASO CLÍNICO}

A evolução da paciente durante as 20 terapias a que foi submetida pode ser observada resumidamente nos quadros 1, 2 e 3 da seção Relato de Caso Clínico, que contêm dados quantificados do tono muscular, atividades funcionais estáticas e atividades funcionais dinâmicas respectivamente. Os dados de atividades funcionais foram colocados em gráficos para a melhor visualização e compreensão.

O quadro 2 que contém a evolução do tono muscular revela que não houve alterações entre a avaliação inicial e a $10^{\underline{a}}$ terapia, mas que entre a $10^{\underline{a}}$ e a $20^{\underline{a}}$ terapia houve diminuição da hipertonia de alguns grupos musculares (músculos rotadores mediais de ombro e músculos extensores de quadril de ambos os hemicorpos). É importante notar que não houve aumento da hipertonia em nenhum grupo muscular. Podemos verificar a evolução da performance motora no gráfico representado na figura 1.

Podemos verificar que a postura em sedestação evoluiu de 4 para 9 graus já na $2^{\mathrm{a}}$ avaliação que corresponde à $10^{\mathrm{a}}$ terapia, significando que além de iniciar a aquisição da postura sem auxílio, já a mantém sem apoio com bom alinhamento de tronco mesmo realizando atividades na postura como manipular objetos. A quadrupedia evoluiu de 8 a 9 graus da avaliação inicial para a $10^{\underline{a}}$ terapia, significando melhora do alinhamento de tronco mesmo ao realizar atividades na postura, o que denota melhora dos ajustes posturais de tronco. A postura ajoeIhada permaneceu estabilizada da avaliação inicial para a $10^{\underline{a}}$ terapia, evoluindo de grau 2 para 5 da $10^{\underline{a}}$ para a $20^{\underline{a}}$ terapia onde adquiriu-se a aquisição da postura sem auxílio de MMSS, sendo estes ainda necessários para a sua manutenção. A postura semi-ajoelhada acompanhou a evolução da postura ajoelhada permanecendo-se es-

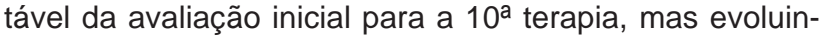
do da $10^{\underline{a}}$ terapia para a $20^{\text {a }}$ terapia do grau 2 para 5 . Verifica-se então que houve incremento do controle de tronco inferior e cintura pélvica na aquisição sem auxílio desta postura. A bipedestação evoluiu com melhora discreta da avaliação inicial para a 10 $0^{\underline{a}}$ terapia, do grau 3 para o grau 4, significando melhora do alinhamento de tronco sendo ainda necessários a aquisição com auxílio de MMSS. Mas verificou-se progressão importante da 10 $10^{\underline{a}}$ terapia para a 20aㅡ terapia, do grau 4 para o grau 9 , concomitantes com a melhora do controle de tronco inferior e de cintura pélvica observados na postura ajoelhada e semi-ajoelhada durante este período, significando uma evolução da aquisição e da manutenção da postura não sendo mais necessárias auxílio de MMSS. A paciente manteve o alinhamento de tronco mesmo ao realizar atividades nesta postura.

Os ganhos relatados nas atividades funcionais estáticas culminaram com a melhora do padrão do engatinhar e a aquisição da marcha como podem ser observados na figura 2 .

O engatinhar evoluiu de grau 2 para 3 , da avaliação inicial para a 10aㅡ terapia, significando a realização da atividade em padrão normal e coordenado sem interferência da hipertonia, realizando-o com aumento da dissociação de cinturas e melhor controle de tronco inferior. A marcha evoluiu de grau 0 para grau 1, da avaliação inicial para a $10^{\underline{a}}$ terapia, significando aquisição da marcha sem apoio apesar de ser realizada em padrão alterada com ausência da dissociação de cinturas e déficit de equilíbrio. Da $10^{\underline{a}}$ para a $20^{\underline{a}}$ terapia a paciente evoluiu do grau 1 para o grau 3, significando a realização da marcha sem apoio em padrão normal e coordenado, sem a interferência da hipertonia, mas ainda com diminuição da dissociação de cinturas e aumento da base de sustentação. Houve melhora significativa do equilíbrio.

\section{CASO CLÍNICO II}

A evolução da paciente durante as 20 terapias a que foi submetida pode ser observada resumidamente nos quadros 1, 2 e 3 da seção Relato de Caso Clínico, que contêm dados quantificados de tono muscular, atividades funcionais estáticas e atividades funcionais dinâmicas respectivamente. Os dados de atividades funcionais foram colocados em gráficos para a melhor visualização e compreensão.

O quadro 1 que contém a evolução do tono muscular revela que não houve alterações entre a avaliação inicial e a $10^{\underline{a}}$ terapia, mas que entre a $10^{\underline{a}}$ e a $20^{\underline{a}}$ terapia houve diminuição da hipertonia de alguns grupos musculares (músculos flexores de punho esquerdo). É importante notar que não houve aumento da hipertonia em nenhum grupo muscular.

Podemos verificar a evolução da performance motora no gráfico representado na figura 3.

Podemos verificar que a postura em sedestação permaneceu-se estável em grau 7 da avaliação inicial para a 10 $0^{\text {a }}$ terapia, observando-se apenas alteração qualitativa da postura com diminuição da inclinação do tronco para 
a esquerda. Da $10^{\underline{a}}$ para a $20^{\underline{a}}$ terapia, observou-se evolução do grau 7 para o grau 8, significando manutenção da postura com alinhamento de tronco, perdendo-a ao realizar atividades na postura. A quadrupedia também manteve-se estável da avaliação inicial para a $10^{\underline{a}}$ terapia com grau 8 , e desta para a $20^{\underline{a}}$ terapia, não havendo melhora do alinhamento de tronco ao realizar atividades na postura. A postura ajoelhada evoluiu do grau 1 para o 2 da avaliação inicial para a $10^{\underline{a}}$ terapia, significando melhora do alinhamento na postura ajoelhada necessitando ainda de auxílio para assumir a postura e de apoio para mantê-la. Da 10aㅡ para a 20 ${ }^{\underline{a}}$ terapia, a criança evoluiu do grau 2 para o grau 7 , significando aquisição da postura sem auxílio, manutenção da mesma sem apoio, mas com alteração do alinhamento de tronco. A postura semiajoelhada permaneceu estabilizada no grau 1 da avaliação inicial para a $10^{\mathrm{a}}$ terapia e só evoluiu da $10^{\mathrm{a}}$ terapia para a 20 a terapia, onde passou do grau 1 para o grau 2 significando melhora do alinhamento de tronco na postura. A bipedestação evoluiu com melhora discreta da avaliação inicial para a $10^{\mathrm{a}}$ terapia, do grau 1 para o grau 2, significando melhora do alinhamento de tronco sendo ainda necessários a aquisição com auxílio e a manutenção com apoio de MMSS. Mas verificou-se progressão importante da $10^{\text {a }}$ terapia para a $20^{\text {a }}$ terapia, do grau 2 para o grau 7, significando uma evolução da aquisição e da manutenção da postura não sendo mais necessárias auxílio de MMSS. O alinhamento de tronco ainda encontra-se alterado.

Os ganhos relatados nas atividades funcionais estáticas culminaram com aquisição da marcha como podem ser observados na figura 4.

O engatinhar permaneceu com padrão normal e coordenado sem a interferência da hipertonia da avaliação inicial para a $20^{\underline{a}}$ terapia. A marcha evoluiu de grau 0 para grau 1 , da avaliação inicial para a $10^{\underline{a}}$ terapia, significando aquisição da marcha com apoio de MMSS apesar de ser realizada em padrão alterada com ausência da dissociação de cinturas e déficit de equilíbrio. Da $10^{\underline{a}}$ para a $20^{\underline{a}}$ terapia a paciente manteve o grau 1 , mas realizou a marcha sem apoio de MMSS ainda com importante déficit de equilíbrio.

Para a realização de uma análise estatística houve a necessidade de agrupar os dados dos dois relatos de casos separados em dois grupos: atividades funcionais estáticas e atividades funcionais dinâmicas. Foi aplicado o teste-t pareado, com a qual verificamos que houve uma melhora significativa da avaliação para a $20^{a}$ terapia nas atividades funcionais estáticas $(p<0.05)$, o mesmo não ocorreu com as atividades funcionais dinâmicas ( $p>0.05)$. Avaliando-se os dados da avaliação inicial para a $10^{\mathrm{a}}$ terapia e desta para a $20^{\text {a }}$ também não foram encontradas diferenças significativas em ambas as atividades, mas observa-se o efeito acumulativo da avaliação inicial para a $20^{\text {a }}$ terapia.

\section{DISCUSSÃO}

Os resultados obtidos com o estudo destes dois casos clínicos são compatíveis com os dados já obtidos em outros estudos de sujeito único realizados com os pacientes com diagnóstico clínico de ECNPI, bem como de outras patologias neurológicas infantis, em atendi- mento fisioterápico no Centro de Docência e Pesquisa do Curso de Fisioterapia da USP $(11,12,13)$.

Em nosso estudo verificou-se que, em ambos os casos, a técnica de FNP incrementou o controle motor nas atividades funcionais estáticas e nas dinâmicas e que os ganhos foram mantidos a cada avaliação. Apesar da diferença ser estatiscamente mais significantes nas atividades funcionais estáticas $(p<0.05)$ não reflete que não haja melhora significante nas atividades funcionais dinâmicas, pois estas estão representadas por um número muito pequeno de atividades oferecendo poucos dados para a análise estatística. Desde já, colocamos a necessidade de aumentar a amostra de sujeitos para averiguar valores mais significativos e, desta forma, criar condições para uma avaliação mais detalhada da evolução de cada atividade.

Com base nos dados já observados, podemos afirmar que estes ganhos adquiridos através da exploração do potencial motor reflexo, automático e voluntário foram retidas pelo processo de aprendizado motor que é dependente da própria execução do movimento através de pistas sensitivas e do repertório reflexo postural básico, da repetição e da plasticidade neural ${ }^{14}$.

Após estudos detalhados de neurofisiologia do ato motor e com resultados significativos de pesquisas realizadas no manejo de pacientes com espasticidade verifica-se que a forma reflexa é o susbstrato para um controle motor normal ${ }^{15}$, todos os procedimentos sugeridos para a facilitação dos padrões gerais promovem aprendizado motor proporcionando "pistas sensoriais" apropriadas ${ }^{16}$, ${ }^{17}$ e a abordagem facilitatória não aumenta o tono muscular dando-nos segurança para verificar os efeitos da aplicação desta técnica no tratamento da criança com ECNPI. Quando um músculo, tanto normal quanto hipertônico, é alongado, há inicialmente um aumento normal da resistência ao movimento. Este aumento é devido aos mecanismos reflexos envolvendo o fuso muscular, mas aos poucos ocorre uma adaptação deste. Nos músculos hipertônicos, a resposta pode ser maior devido aos desequilíbrios pré-existentes, mas a adaptação também ocorre podendo retornar até mesmo a um tono menor que o inicial. Então, este abalo encontrado em pacientes com síndromes de liberação não devem ser considerado uma "piora do quadro tônico". Se isto for aplicado em intensidade e freqüência regulares ocorre um aprendizado através do mecanismo de habituação provocando maior adaptação do fuso ${ }^{18}$. Isto já foi comprovado em um estudo com adultos hemiplégicos onde exercícios envolvendo carga e facilitação, geravam uma alteração apenas transitória do tono muscular ${ }^{19}$. Foi demonstrado também que um programa de treinamento de resistência em crianças com ECNPI espástica pode aumentar a força do músculo isolado, eliminando a preocupação que exercícios de resistência pesada poderia ativar atividade muscular indesejável ${ }^{20}$.

A eficácia da intervenção facilitatória em crianças com ECNPI já foi relatada em dois estudos. O primeiro, um relato de caso único de uma criança do sexo masculino de 4 anos de idade que objetivava avaliar quantitativamente o tono muscular, performance motora e alinhamento postural geral em sedestação e qualitativamente a utilização práxica das mãos e freqüência na motricida- 
de voluntária espontânea, onde verificou-se após 100 sessões terapêuticas um ganho quantitativo e qualitativo na performance motora sem alteração do tono muscu$\operatorname{lar}^{11}$. O segundo, que objetivava a avaliação e quantificação de uma possível melhora da motricidade de MMSS em duas crianças com ECNPI, tipo diplegia espástica, sexo masculino, com cinco e sete anos de idade através de intervenção baseada nas técnicas de FNP durante 18 terapias, onde observou-se que não houve alteração do tono muscular nas duas crianças, mas ocorreu alterações nas atividades funcionais (sedestação, quadrupedia, ajoelhado e deslocamento a partir da quadrupedia) revelando melhora do controle de tronco e dos ajustes posturais estáticos e dinâmicos ${ }^{12}$.

Em ambos os casos o padrão de aquisição motora não foi acompanhado de aumento do tono muscular, pelo contrário, acompanhando o desenvolvimento das atividades funcionais da avaliação inicial para a décima terapia não observou-se alteração alguma do tono muscular relativo a todos os grupos musculares havendo mesmo diminuição do tono muscular após a $10^{\underline{a}}$ terapia, contrariando o que se costuma afirmar que este tipo de abordagem aumentaria o tono muscular, pois o fuso muscular possui capacidade de adaptação a diferentes níveis de demanda através do processo de habituação ${ }^{11,13}$. Portanto, se ocorre certo aumento do tono muscular no momento em que é realizado um estiramento, esta alteração é apenas transitória ${ }^{13,19}$. Portanto, observou-se que o incremento motor no controle de tronco e conseqüente liberação das atividades distais dos membros, que ocorreu de forma mais intensa da décima para a vigésima terapia, foi concomitante com a diminuição do tono muscular de alguns grupos musculares principalmente de MMSS que são as regiões menos acometidas na diparesia espástica. Torna-se importante ressaltar a não utilização de medicamento algum com efeito anti-espástico por ambas as pacientes durante o período de tratamento ou mesmo antes.

Em ambos os casos verificou-se que a sedestação foi a postura que evolui mais rapidamente pois necessita de menor controle pélvico ou de MMII e que o melhor controle de tronco superior que acompanhou a evolução desta postura foi evidente em outras posturas como quadrupedia e bipedestação. As posturas ajoelhada e semi-ajoeIhada foram as que mostraram evolução mais lenta em ambos os casos, principalmente da avaliação inicial para a décima terapia, pois necessitam de maior controle de tronco inferior, cintura pélvica e em menor grau de MMII.
Acompanhando a melhor evolução destas da décima para a vigésima terapia observou-se um importante incremento motor postural na bipedestação. A bipedestação acompanhou o conjunto de maiores incrementos tanto de fortalecimento de musculatura abdominal e extensora como de controle de tronco superior, tronco inferior, cinturas e de membros, principalmente da décima para a vigésima terapia.

O engatinhar foi a atividade funcional entre as dinâmicas e estáticas que menos apresentou alterações na avaliação inicial evoluindo para um padrão normal e coordenado nas primeiras terapias em ambas as pacientes. A marcha apresentou melhora significativa da avaliação inicial para a décima terapia em ambas as pacientes pois passaram da não realização para a marcha realizada em padrão alterado. É importante notificar que esta foi realizada sem apoio de MMSS pela paciente do caso 1, o que não ocorreu com a paciente do caso 2 que apresentou este tipo de marcha somente com apoio de MMSS em uma superfície mais elevada. Pode parecer, então, que a paciente do caso 2 não tenha evoluído na escala da marcha da décima para a vigésima terapia pois manteve o grau 1 de realização da marcha em padrão alterado, mas neste período esta foi realizado já sem apoio de MMSS. Então da décima para a vigésima terapia, ambas as pacientes continuaram a evolução no padrão da marcha.

\section{CONCLUSÃO}

Os dados obtidos neste estudo permite-nos observar que os protocolos para avaliação de tono muscular e de atividades funcionais estáticas e dinâmicas servem-nos de instrumentos de grande valia para quantificar o quadro motor dos pacientes de forma única de modo que possam ser realizadas análises intra e inter sujeito de diversos procedimentos fisioterápicos ou mesmo clínicos.

Através dos resultados obtidos por eles foi possível observar que o procedimento fisioterapêutico de FNP levaram ambas as pacientes a melhoras quantitativas em relação às atividades funcionais estáticas e dinâmicas.

A melhora do quadro tônico influenciado pelo incremento do controle motor na realização das atividades funcionais observado em ambas as pacientes denota a importância da utilização de estratégias terapêuticas que envolvam facilitação nos moldes preconizados pela técnica de FNP, devendo ser considerada entre as opções no tratamento da Encefalopatia Crônica não Progressiva da Infância. 


\section{REFERÊNCIASBIBLIOGRÁFICAS}

1. Diament, A. Exame neurológico do lactente. In: Diament, A.; Cypel, S., org. Neurologia infantil. São Paulo: Atheneu, 1996.

2. Leonard, C.T.; Hirschfeld, H.; Moritani, T.; Forssberg, H.. Myotatic Reflex Development in Normal Children and Children with Cerebral Palsy. Experimental Neurology. 111, p. 379-82, 1991.

3. Vecchierini-Blineau, M.F.; Guiheneuc, P. Excitability of the Monosynaptic Reflex Pathway in the Child from Birth to Four Years of Age. Journal of Neurology, Neurosurgery, and Psychiatry. 44, p. 309-14, 1991.

4. Halpern, D. Reabilitação de crianças com lesão cerebral. In: Kottke, J.; Lehmann, J.F., org. Tratado de medicina física e reabilitação de Krusen. São Paulo: Manole, 1994.

5. Bobath, B.; Bobath, K. Desenvolvimento motor nos diferentes tipos de Paralisia Cerebral. São Paulo: Manole, 1978.

6. Tiroshi, E. Rabino, S. Physiotheraphy for children with cerevral palsy. The American Journal of diseases of children, v. 143, n.5, p.552-5, may, 1989.

7. O'Sullivan, S.B. Estratégias para o incremento do controle motor. In: O'Sullivan, S.B.; Schmitz, T.J., org. Fisioterapia. Avaliação e tratamento. São Paulo: Manole, 1993.

8. Durigon, O.F.S; Piemonte, M.E.P.. Desenvolvimento de Protocolo para avaliação do Tono Muscular. In: Congresso Brasileiro de Fisioterapia, 9. e Congresso Paulista de Fisioterapia, 4., 1993, São Paulo. Anais. São Paulo: ABF, 1993. p. 31.

9. Durigon, O.F.S; SÁ, C.S.C.; Sita, L.V.. Validação de um protocolo de Avaliação do Desempenho Motor e Funcional de Crianças com Paralisia Cerebral. In: XIII Congresso Nacional de Paralisia Cerebral, São Paulo, 1998. Anais.

10. Durigon, O.F.S; SÁ, C.S.C.; Sita, L.V.. Desenvolvimento de um protocolo de avaliação do desempenho motor e funcional de crianças com Paralisia Cerebral. Arquivos de Neuro-Psiquiatria, 1996.

11. Durigon, O.F.S; Sá, C.S.C.. Intervenção fisioterápica facilitatória em paciente com encefalopatia não progressiva crônica da infância. Revista de Fisioterapia da Universidade de São Paulo, v. 3, n. 1/2, p. 54-64, jan./dez., 1996.

12. Sá, C.S.C.; Carbonário, F.. Intervenção Fisioterápica na Motricidade de Membros Superiores de Crianças com Paralisia Cerebral. Revista Brasileira de Fisioterapia. Suplemento Especial, p. 83-4, outubro, 1998.

13. Sá, C.S.C, Mudanças motoras, sensoriais e cognitivas em crianças com paralisia cerebral espástica diparéticas submetidas a intervenão fisioterápica pelas abordagens Kabat e Bobath. Dissertação de Mestrado - IP/USP, 1999.

14. Kandel, E.R.; Schwartz, J.H., Jessel T.M.. Principles of Neural Science. Pretnice -Hall International $4^{\text {th }}, 2001$.

15. Shumway-Cook, A.; Woolla, M.H.. Motor Control - Theory and Practical Applications. Baltimore, Ed. Willians \& Wilkins, 2001.

16. Voss,D.E.; Ionta, M.K.; Myers, B.J.. Facilitação neuromuscular proprioceptiva. São Paulo: Panamericana, 1987.

17. Adler,S.S.; Beckers, D. Buck, M. PNF- Facilitação Neuromuscular proprioceptiva. ed. Manole, 1999.

18. Durigon, O.F.S. Alongamento muscular. Parte I- A interação neuromuscular. Revista de Fisioterapia da Universidade de São Paulo, v.2, n. 1, p.40-4, jan./jul., 1995.

19. Durigon, O.F.S; Souza Jr., J.A.. Comparação de dois procedimentos: alongamento e carga no manejo da espasticidade em hemiplégicos. In: Reunião anual da Sociedade Brasileira para o Progresso da Ciência, 46., Vitória, 1994. Anais. p. 144.

20. Damiano, D.L.; Vaughan, C.L.; Abel, M.F.. Muscle response to heavy resistance exercise in children with spastic cerebral palsy. Dev Med Child neurol, v. 37, n. 8, p. 731-739, aug, 1995. 